\title{
REPRODUCIBILIDAD DEL ESTUDIO DE LA CABEZA DEL NERVIO ÓPTICO Y DE LA CAPA DE FIBRAS NERVIOSAS MEDIANTE TOMOGRAFÍA ÓPTICA DE COHERENCIA
}

\author{
REPRODUCIBILITY OF OPTIC NERVE HEAD AND RETINAL \\ NERVE FIBER LAYER THICKNESS MEASUREMENTS USING \\ OPTICAL COHERENCE TOMOGRAPHY
}

\author{
PUEYO $\mathrm{V}^{1}$, POLO $\mathrm{V}^{2}$, LARROSA JM ${ }^{2}$, MAYORAL $\mathrm{F}^{1}$, FERRERAS A ${ }^{2}$, HONRUBIA FM ${ }^{2}$
}

\begin{abstract}
RESUMEN
Objetivo: Evaluar la reproducibilidad de la medición del espesor de la capa de fibras nerviosas de la retina y de la cabeza del nervio óptico mediante tomografía óptica de coherencia (OCT).

Métodos: Se incluyeron 32 sujetos normales, 41 hipertensos oculares y 33 pacientes glaucomatosos. Los grupos de estudio se establecieron según sus niveles de presión intraocular, evaluación de estereo-fotografías papilares y resultados de perimetrías automatizadas. La evaluación de la CFNR y nervio óptico se realizó mediante OCT (OCT III, Zeiss Instruments). Se realizaron 3 exploraciones de cada ojo. Se calculó el coeficiente de variación de cada uno de los parámetros en los diferentes grupos del estudio y se compararon los resultados obtenidos entre los tres grupos.

Resultados: El espesor medio de la CFNR evidenciaron la mejor reproducibilidad con un $\mathrm{CV}$ de $5,9 \%$. El cuadrante inferior y los sectores correspondientes a las 10, 6 y 7 horas han mostrado los mejores $\mathrm{CV}(6,3 \%, 7,4 \%, 7,5 \%$ y $8,6 \%$ respectivamente). Con respecto a los parámetros papilares los cocientes excavación/disco vertical, horizontal y el
\end{abstract}

\begin{abstract}
Purpose: To evaluate the reproducibility of repeated quantitative measurements of optic nerve head topography and retinal nerve fiber layer thickness. Methods: This study included 32 normal subjects, 41 patients with ocular hypertension and 33 patients with glaucoma. The study groups were defined by the intraocular pressure levels, optic disc stereophotographs and perimetric performance. Optic disc and retinal nerve fiber layer assessments were performed by ocular coherence tomography (OCT III, Zeiss Instruments). OCT examination was repeated three times in each subject and the coefficient of variability was elaborated for each parameter and group of the study, and compared among them.

Results: The average retinal nerve fiber layer thickness measurement showed the best reproducibility figures with a coefficient of variability of $5.9 \%$. The inferior quadrant and the 10, 6 and 7 clock-hours (coefficients of variability $6.3 \%, 7.4 \%, 7.5 \%$ and $8.6 \%$ respectively) showed higher reproducibility. The optic nerve head assessment showed the best reproducibility for the cup-to-disc ratios (vertical, horizontal and area ratios), with a coefficient of
\end{abstract}

Recibido: 6/5/05. Aceptado: 11/4/06

Servicio de Oftalmología. Hospital Universitario Miguel Servet. Zaragoza. España.

1 Licenciado en Medicina.

2 Doctor en Medicina.

Correspondencia:

Victoria Pueyo

Servicio de Oftalmología. Hospital Universitario Miguel Servet

Paseo Isabel la Católica, 1-3

50009 Zaragoza

España

E-mail: vicpueyo@hotmail.com 
cociente área de excavación/papila mostraron la mayor reproducibilidad, con coeficientes de variación de $5,0 \%, 4,2 \%$ y $6,7 \%$, respectivamente. A pesar de apenas existieron diferencias estadísticamente significativas entre los grupos, los pacientes con glaucoma presentaron coeficientes de variación superiores a los otros dos grupos.

Conclusiones: Las mediciones del espesor de la CFNR y parámetros morfométricos del nervio óptico obtenidos mediante OCT resultan reproducibles confirmando la utilidad de esta técnica para el diagnóstico y seguimiento del paciente hipertenso ocular, encontrándose ciertas limitaciones en algunos parámetros en pacientes glaucomatosos.

Palabras clave: Glaucoma, reproducibilidad, OCT, capa de fibras nerviosas. variability of $5.0 \%, 4.2 \%$ and $6.6 \%$ respectively. Although differences among groups were barely statistically significant, the glaucoma group showed coefficients of variability higher than the other two groups.

Conclusions: Optic nerve head analysis and retinal nerve fiber layer thickness can be reproducibly assessed by OCT. The reproducibility of the device supports its potential use for ocular hypertension follow-up, although its use in glaucoma patients may present difficulties in some parameters (Arch Soc Esp Oftalmol 2006; 81: 205-212).

Key words: Glaucoma, reproducibility, OCT, nerve fiber layer.

\section{INTRODUCCIÓN}

El glaucoma crónico simple representa una neuropatía óptica progresiva caracterizada por la existencia de alteraciones morfológicas y estructurales a nivel del nervio óptico y de la capa de fibras nerviosas de la retina (CFNR) así como la aparición de defectos funcionales evaluados principalmente a través de la exploración del campo visual (1-5).

Clásicamente el estudio de la CFNR y nervio óptico se ha basado en la exploración funduscópica y fotográfica convencionales, limitadas por la variabilidad y subjetividad dependiente del observador. El reciente desarrollo de técnicas de imagen digital ha supuesto un avance importante para la valoración de los cambios estructurales que se producen en la enfermedad glaucomatosa, con la aparición de instrumentos diagnósticos objetivos y cuantitativos.

La tomografía óptica de baja coherencia (OCT) representa un nuevo método de evaluación de la CFNR y nervio óptico caracterizado por su rapidez y sencillez, que permite obtener mediciones del espesor de la CFNR. Los estudios preliminares realizados con esta técnica han evidenciado su utilidad en la práctica clínica para detectar las pérdidas o reducciones en la CFNR que se producen en el glaucoma (6-11).

El objetivo del presente estudio fue evaluar la reproducibilidad de las mediciones repetidas del espesor de la capa de fibras nerviosas de la retina (CFNR) y parámetros morfométricos papilares eva- luada mediante tomografía óptica de coherencia (OCT) en sujetos normales, hipertensos oculares y glaucomatosos.

\section{SUJETOS, MATERIAL Y MÉTODO}

\section{Sujetos}

Se han incluido en el presente estudio un total de 32 sujetos normales, 41 hipertensos oculares y 33 pacientes glaucomatosos revisados en el Servicio de Oftalmología de nuestro centro.

Los criterios de inclusión en el estudio fueron: edad entre 30 y 80 años, agudeza visual mayor o igual a 8/10 (escala de Snellen), defectos de refracción menor a 5 dioptrías esféricas y astigmatismo menor de 3 dioptrías y medios oculares transparentes.

Se excluyeron aquellos sujetos con antecedentes de traumatismos o cirugía oculares, enfermedades sistémicas con repercusión oftálmica o incapacidad para realizar alguna de las pruebas incluidas en el protocolo de estudio.

Se realizó una exploración oftalmológica completa a todos los sujetos, incluyendo biomicroscopía con lámpara de hendidura, medición de presión intraocular (PIO) mediante tonometría de aplanación, esterofotografía papilar, perimetría automatizada convencional y evaluación de la capa de fibras de la retina (CFNR) y parámetros morfométricos papilares 
mediante tomografía óptica de coherencia (OCT 3000; Humphrey Zeiss Instruments, Dublín, CA).

Se solicitó consentimiento informado de todos los sujetos incluidos. Sólo se incluyó un ojo de cada paciente en el estudio y en caso de que ambos ojos fueran elegibles se seleccionó uno de ellos al azar (cara o cruz de moneda).

La perimetría automatizada convencional se realizó mediante un perímetro Humphrey Field Analyzer y programa 30-II, STATPAC 2. Los defectos perimétricos fueron definidos por: grupo de al menos 3 puntos alterados con un nivel de probabilidad menor a $5 \%$ o grupo de al menos 2 puntos alterados con un nivel de probabilidad menor a $1 \%$ (se excluyen los puntos situados en la corona periférica o en los polos de la mancha ciega) y/o índices globales (MD o CPSD) con pérdidas con un nivel de probabilidad menor al $2 \%$ y/o prueba de hemicampo de glaucoma fuera de límites normales. Para considerar una perimetría como patológica fue necesaria la presencia de defectos compatibles con daño glaucomatoso, reproducibles, presentes en al menos tres exploraciones.

La neuropatía óptica glaucomatosa (NOG) fue definida por la presencia de adelgazamiento del anillo neurorretiniano, presencia de muescas, hemorragias papilares y /o asimetría interocular de los cocientes excavación/papila mayores de 2/10, mediante valoración de estereofotografías.

Los sujetos normales (grupo I) fueron definidos como aquellos con PIO menor de $21 \mathrm{~mm}$ de $\mathrm{Hg}$ y nervio óptico y perimetría automatizada convencional normales. Los hipertensos oculares (grupo II) se definieron por PIO mayor o igual a $21 \mathrm{~mm}$ de $\mathrm{Hg}$, nervio óptico sin signos de neuropatía óptica glaucomatosa y ausencia de defectos perimétricos. Los sujetos glaucomatosos (grupo III) fueron definidos por PIO mayor o igual a $21 \mathrm{~mm}$ de $\mathrm{Hg}$, neuropatía óptica glaucomatosa y perimetría automatizada convencional alterada.

\section{Tomografía de baja coherencia óptica}

La evaluación de los espesores de la CFNR y parámetros morfométricos papilares fue realizada mediante la versión comercial del OCT 3000 (Humphrey-Zeiss Instruments).

Tras dilatación pupilar con tropicamida 1\% (Tropicamida $1 \%{ }^{\circledR}$ AlconCusí, El Masnou, Barcelona, España) se realizaron tres exploraciones (separadas unos minutos en el tiempo) empleando un protocolo de barrido óptico circular de 3,4 $\mathrm{mm}$ de diámetro centrado en el disco óptico (Protocolo de Grosor de la CFNR - RNFL Thickness-). Este protocolo permite obtener las siguientes mediciones de la CFNR: espesor medio de la CFNR $\left(360^{\circ}\right)$, espesor en los 4 cuadrantes retinianos y espesores en cada segmento horario (30 grados por cada posición horaria). Adicionalmente obtenemos el resultado de diferentes parámetros de la OCT (Imax/Smax, Smax/Imax, Smax/Tavg, Imax/Tavg, Smax/Navg, Max-Min, Smax, Imax, Savg e Iavg).

Posteriormente se realizaron tres exploraciones de la cabeza del nervio óptico (separadas unos minutos en el tiempo) empleando un patrón de barrido de 6 líneas radiales de $4 \mathrm{~mm}$ equidistantes entre sí a través de un eje central común (Protocolo de Disco óptico - Optic Disc-). A partir de los barridos lineales el protocolo de análisis aporta los resultados cuantitativos de los siguientes parámetros morfométricos de la cabeza del nervio óptico: Área vertical integrada del Anillo -Volumen-, Anchura horizontal integrada del Anillo -Área-, Área del disco, Área de la excavación, Área del Anillo, Cociente de área excavación/disco, Cociente de área excavación/disco horizontal y Cociente de área excavación/disco vertical.

En caso de que la OCT no identificara correctamente los límites del epitelio pigmentario a la hora de realizar las medidas de la papila, el propio examinador corrigió dichos límites manualmente.

\section{Análisis estadístico}

La exploración del espesor de la CFNR y de los parámetros morfométricos papilares fue repetida en 3 ocasiones para cada sujeto, recolocando al paciente entre cada una de las exploraciones.

Las diferentes variables obtenidas fueron introducidas en una hoja de cálculo (Excel para Windows) y se determinaron la media, desviación estándar y coeficiente de variación (CV) de cada parámetro. La variabilidad de cada parámetro se determinó como la media de los coeficientes de variación en cada uno de los sujetos, considerándose reproducibles aquellos parámetros con coeficiente de variación menores al 10\% y altamente reproducibles aquellos con coeficiente de variación menor al 5\%. Este estudio se calculó tanto en el total de sujetos como en cada uno de los tres grupos 
de estudio. Por último, se realizó una comparación de los coeficientes de variación obtenidos en cada uno de los grupos en todos los parámetros analizados, mediante un análisis de sus varianzas (ANOVA).

\section{RESULTADOS}

Se han incluido en el presente estudio un total de 106 ojos de 106 sujetos: 32 sujetos normales, 41 hipertensos oculares y 33 glaucomatosos, cuyas características descriptivas se recogen en la tabla I.

En relación a los espesores de la CFNR los resultados del estudio quedan reflejados en la tabla II. El parámetro que mejor reproducibilidad mostró fue el espesor global de la CFNR, que obtuvo un valor de coeficiente de variación del $5,9 \%$. Con respecto a los cuadrantes retinianos, el cuadrante inferior fue el más reproducible (CV del 6,3\%), seguido del temporal (CV del 7,2\%), nasal (CV del 9,7\%) y superior (CV del 10,5\%). En el análisis por sectores horarios las horas que presentaron unos menores valores de $\mathrm{CV}$ fueron los correspondientes a las 10 , 6 y 7 horas (CV del 7,4\%, 7,5\% y $8,6 \%$ respectivamente).

La tabla III muestra los coeficientes de variación de los parámetros de la OCT. La mejor reproducibilidad se detectó en el grosor promedio de la CFNR en el

Tabla I. Características descriptivas de las poblaciones incluidas en el estudio

\begin{tabular}{|c|c|c|c|c|c|c|c|}
\hline \multirow[b]{3}{*}{ Edad } & \multicolumn{2}{|c|}{ GRUPO I } & \multicolumn{3}{|c|}{ GRUPO II } & \multicolumn{2}{|c|}{ GRUPO IV } \\
\hline & Media (DE) & \multirow{2}{*}{$\frac{p}{3}$} & \multicolumn{2}{|c|}{ Media (DE) } & \multirow[b]{2}{*}{3} & Media (DE) & \multirow{2}{*}{$\frac{\mathrm{p}}{1,2}$} \\
\hline & $60,6 \quad(7,2)$ & & 53,6 & $(11,8)$ & & $62,4 \quad(10,9)$ & \\
\hline Paquimetría & $560,4 \quad(21,8)$ & - & 517,7 & $(42,8)$ & - & $550,9 \quad(44,7)$ & - \\
\hline AV & $0,85 \quad(0,1)$ & - & 0,91 & $(0,1)$ & - & $0,86 \quad(0,1)$ & - \\
\hline PIO basal & $14,6 \quad(2,3)$ & 2,3 & 22,4 & $(1,4)$ & 1 & $23,3 \quad(2,7)$ & 1 \\
\hline Cociente E/D & $1,08 / 5$ & 2,3 & $1,8 /$ & & 1,3 & $3,4 / 5$ & 1,2 \\
\hline $\mathrm{DM}$ & $-0,56 \quad(1,4)$ & 3 & $-0,49$ & $(1,31)$ & 3 & $-5,7 \quad(6,9)$ & 1,2 \\
\hline CPSD & $1,1 \quad(0,9)$ & 3 & 1,2 & $(1,1)$ & 3 & $(4,1)$ & 1,2 \\
\hline Area disco & $2,23 \quad(0,4)$ & - & 2,18 & $(0,3)$ & - & $(0,3)$ & - \\
\hline
\end{tabular}

(1) Diferencias estadísticamente significativas con respecto al grupo control; (2) Diferencias estadísticamente significativas con respecto al grupo de hipertensos oculares; (3) Diferencias estadísticamente significativas con respecto al grupo de glaucoma.

Tabla II. Valores de las medias y desviaciones estándar (DE) de los coeficientes de variación de los espesores de la CFNR por cuadrantes y sectores horarios en valores totales, sujetos normales (grupo I), hipertensos oculares (grupo II) y glaucomas (grupo III)

\begin{tabular}{|c|c|c|c|c|c|c|c|c|}
\hline & \multicolumn{2}{|c|}{ TOTALES } & \multicolumn{2}{|c|}{ GRUPO I } & \multicolumn{2}{|c|}{ GRUPO II } & \multicolumn{2}{|c|}{ GRUPO IV } \\
\hline & $\begin{array}{l}\text { Media } \\
\text { de CV }\end{array}$ & $\begin{array}{c}\text { D E } \\
\text { de CV }\end{array}$ & $\begin{array}{l}\text { Media } \\
\text { de CV }\end{array}$ & $\begin{array}{c}\text { D E } \\
\text { de CV }\end{array}$ & $\begin{array}{l}\text { Media } \\
\text { de CV }\end{array}$ & $\begin{array}{c}\text { D E } \\
\text { de CV }\end{array}$ & $\begin{array}{l}\text { Media } \\
\text { de CV }\end{array}$ & $\begin{array}{c}\text { D E } \\
\text { de CV }\end{array}$ \\
\hline H1 & 12,71 & 21,74 & 9,56 & 3,74 & 9,00 & 6,33 & 22,37 & 38,25 \\
\hline $\mathrm{H} 2$ & 13,36 & 20,05 & 9,72 & 2,56 & 10,10 & 8,07 & 20,68 & 35,12 \\
\hline H3 & 11,37 & 9,84 & 11,60 & 11,38 & 7,18 & 4,71 & 15,84 & 15,29 \\
\hline H4 & 9,88 & 12,03 & 11,47 & 5,88 & 7,30 & 4,08 & 15,81 & 20,37 \\
\hline H5 & 8,69 & 12,20 & 7,63 & 6,79 & 6,13 & 4,28 & 15,13 & 20,55 \\
\hline H6 & 7,53 & 8,62 & 4,35 & 3,16 & 8,27 & 7,18 & 10,41 & 13,31 \\
\hline H7 & 8,60 & 17,09 & 5,33 & 3,11 & 7,95 & 10,55 & 15,20 & 29,03 \\
\hline H8 & 9,37 & 13,93 & 7,69 & 6,94 & 6,67 & 5,32 & 16,47 & 23,86 \\
\hline H9 & 9,86 & 14,40 & 5,81 & 3,61 & 7,55 & 4,26 & 16,47 & 25,16 \\
\hline H10 & 7,37 & 14,00 & 6,43 & 4,38 & 4,53 & 3,19 & 13,41 & 25,01 \\
\hline H11 & 7,65 & 14,13 & 6,18 & 5,76 & 5,46 & 3,18 & 14,19 & 25,02 \\
\hline H12 & 10,39 & 17,51 & 8,98 & 5,09 & 5,62 & 3,31 & 20,29 & 30,27 \\
\hline Superior & 10,55 & 20,79 & 8,65 & 4,07 & 4,97 & 3,17 & 17,76 & 31,25 \\
\hline Inferior & 6,26 & 10,78 & 2,91 & 3,20 & 6,10 & 5,76 & 10,53 & 18,48 \\
\hline Nasal & 9,73 & 10,31 & 8,75 & 2,85 & 6,83 & 4,66 & 15,25 & 16,68 \\
\hline Temporal & 7,28 & 13,91 & 5,79 & 2,29 & 4,74 & 3,22 & 13,20 & 24,93 \\
\hline Espesor medio CFNR & 5,86 & 9,45 & 6,04 & 3,76 & 4,01 & 2,22 & 9,59 & 16,26 \\
\hline
\end{tabular}


Estudio de reproducibilidad de la tomografía óptica de coherencia

Tabla III. Valores de las medias y desviaciones estándar (DE) de los coeficientes de variación de los cocientes de la OCT en valores totales, sujetos normales (grupo I), hipertensos oculares (grupo II) y glaucomas (grupo III)

\begin{tabular}{|c|c|c|c|c|c|c|c|c|}
\hline & \multicolumn{2}{|c|}{ TOTALES } & \multicolumn{2}{|c|}{ GRUPO I } & \multicolumn{2}{|c|}{ GRUPO II } & \multicolumn{2}{|c|}{ GRUPO IV } \\
\hline & $\begin{array}{l}\text { Media } \\
\text { de CV }\end{array}$ & $\begin{array}{c}\text { D E } \\
\text { de } C V\end{array}$ & $\begin{array}{l}\text { Media } \\
\text { de CV }\end{array}$ & $\begin{array}{c}\text { D E } \\
\text { de } C V\end{array}$ & $\begin{array}{l}\text { Media } \\
\text { de CV }\end{array}$ & $\begin{array}{c}\text { D E } \\
\text { de CV }\end{array}$ & $\begin{array}{l}\text { Media } \\
\text { de CV }\end{array}$ & $\begin{array}{c}\text { D E } \\
\text { de CV }\end{array}$ \\
\hline Imax/Smax & 10,86 & 19,53 & 4,79 & 4,03 & 8,91 & 10,92 & 24,78 & 36,27 \\
\hline Smax/Imax & 12,20 & 23,62 & 5,06 & 3,97 & 10,04 & 13,68 & 29,07 & 43,72 \\
\hline Smax/Tavg & 11,61 & 17,36 & $8,01(*)$ & 5,16 & $7,48(*)$ & 6,49 & $26,87(*)$ & 31,26 \\
\hline Imax/Tavg & 11,14 & 22,29 & 6,43 & 6,80 & 7,16 & 10,33 & 26,23 & 42,53 \\
\hline Smax/Navg & 11,93 & 19,43 & 12,01 & 10,04 & 7,43 & 3,30 & 22,02 & 38,27 \\
\hline Max-Min & 8,21 & 11,53 & 6,37 & 7,61 & 7,11 & 6,81 & 13,56 & 21,54 \\
\hline Smax & 8,65 & 16,18 & 6,00 & 6,02 & 5,12 & 3,80 & 21,33 & 30,50 \\
\hline Imax & 7,50 & 14,13 & 4,01 & 3,63 & 7,09 & 9,82 & 15,86 & 26,27 \\
\hline Savg & 9,53 & 19,94 & 6,64 & 2,69 & 3,58 & 1,97 & 25,93 & 37,56 \\
\hline Iavg & 6,93 & 12,09 & 2,67 & 2,71 & 7,63 & 6,54 & 14,08 & 22,97 \\
\hline
\end{tabular}

*: En este parámetro se observaron diferencias estadísticamente significativas $(\mathrm{p}<0,05)$ entre el grupo de glaucomas y los otros dos grupos analizados.

cuadrante inferior - Iavg - (CV del 6,9\%), seguido del grosor máximo en el cuadrante inferior - Imax$(\mathrm{CV}$ del $7,5 \%)$ y cuadrante superior $-\mathrm{Smax}-(\mathrm{CV}$ del $8,6 \%$ ).

En referencia al estudio de los parámetros morfológicos de la cabeza del nervio óptico se evidenció que los índices excavación-disco: Índice horizontal excavación-disco - CD hor ratio-, Índice vertical excavación-disco - CD ver ratio- e Índice de área excavación-disco $-\mathrm{CD}$ area ratio- $(\mathrm{CV}$ del 4,2\%, $5,0 \%$ y $6,7 \%$ respectivamente), mostraron los mejores valores de reproducibilidad (tabla IV).

Como se observa en las tablas II, III y IV, existieron diferencias entre los coeficientes de variación hallados en cada uno de los grupos de estudio. De este modo, se detectó que la mayoría de los parámetros evaluados se mostraron reproducibles en los grupos I (normales) y II (hipertensos oculares), presentando coeficientes de variación inferiores al 10\%
30 de los 37 parámetros en el grupo de sujetos normales y 32 de los 37 en el grupo de hipertensos oculares. En cambio, el grupo de pacientes con glaucoma mostró coeficientes de variación claramente superiores a los anteriores, siendo en su mayoría superiores al 10\% (31 de los 37 parámetros analizados). El parámetro que mejor reproducibilidad presentó en el grupo de pacientes con glaucoma fue el espesor medio de la CFNR (CV 9,59\%).

Con el objeto de analizar las diferencias existentes entre los tres grupos de estudio en cuanto a sus coeficientes de variación, se realizó una comparación de los mismos mediante un análisis de la varianza (ANOVA). A pesar de que se observó una clara tendencia a presentar coeficientes de variación superiores en el grupo de pacientes con glaucoma, respecto a los otros dos grupos analizados, estas diferencias sólo fueron estadísticamente significativas $(p<0,05)$ en el parámetro Smax/Tavg.

Tabla IV. Valores de las medias y desviaciones estándar (DE) de los coeficientes de variación de los parámetros de la cabeza del nervio óptico en valores totales, sujetos normales (grupo I), hipertensos oculares (grupo II) y glaucomas (grupo III)

\begin{tabular}{|c|c|c|c|c|c|c|c|c|}
\hline & \multicolumn{2}{|c|}{ TOTALES } & \multicolumn{2}{|c|}{ GRUPO I } & \multicolumn{2}{|c|}{ GRUPO II } & \multicolumn{2}{|c|}{ GRUPO IV } \\
\hline & $\begin{array}{l}\text { Media } \\
\text { de CV }\end{array}$ & $\begin{array}{c}\text { D E } \\
\text { de CV }\end{array}$ & $\begin{array}{l}\text { Media } \\
\text { de CV }\end{array}$ & $\begin{array}{c}\text { D E } \\
\text { de CV }\end{array}$ & $\begin{array}{l}\text { Media } \\
\text { de CV }\end{array}$ & $\begin{array}{c}\text { D E } \\
\text { de CV }\end{array}$ & $\begin{array}{l}\text { Media } \\
\text { de CV }\end{array}$ & $\begin{array}{c}\text { D E } \\
\text { de CV }\end{array}$ \\
\hline Área vert integrada (vol) & 21,60 & 17,05 & 26,47 & 19,41 & 12,96 & 5,92 & 25,97 & 17,60 \\
\hline Ánchura horiz integrada (area) & 9,13 & 6,34 & 11,27 & 10,37 & 7,84 & 6,04 & 11,91 & 2,39 \\
\hline Área de disco & 8,03 & 4,38 & 9,27 & 6,27 & 5,47 & 3,34 & 9,37 & 1,87 \\
\hline Área de excavación & 8,34 & 7,24 & 6,90 & 5,16 & 10,78 & 12,31 & 6,32 & 4,77 \\
\hline Área de anillo & 15,16 & 11,45 & 18,09 & 18,83 & 7,95 & 6,24 & 18,50 & 3,85 \\
\hline Cociente área E/D & 6,68 & 5,07 & 6,11 & 3,54 & 8,53 & 9,17 & 7,53 & 2,84 \\
\hline Cociente área E/D horizontal & 4,22 & 3,32 & 4,61 & 2,26 & 5,35 & 4,70 & 4,52 & 3,80 \\
\hline Cociente área E/D vertical & 5,00 & 4,01 & 4,41 & 1,84 & 6,48 & 6,28 & 5,38 & 4,75 \\
\hline
\end{tabular}




\section{DISCUSIÓN}

La tomografía óptica de baja coherencia es un instrumento con alta resolución de imagen en el análisis de la capa de fibras nerviosas de la retina y la cabeza del nervio óptico, cuyas mediciones no se ven afectadas por patología refractiva ni cataratas incipientes o moderadas (12). Desde el punto de vista práctico para que un instrumento diagnóstico pueda considerarse de utilidad en la clínica debe garantizar una alta reproducibilidad. En este sentido, diversos estudios han valorado la posibilidad de aumentar el número de barridos con el fin de disminuir la variabilidad de sus mediciones (13), recomendando la exploración mediante series de cinco análisis (14).

En nuestro estudio hemos obtenido una elevada reproducibilidad en las mediciones de los espesores de la CFNR, así como de los parámetros morfométricos papilares, resultados coincidentes con los confirmados previamente por diversos autores (1519). Así Blumenthal et al (15) refirieron el cuadrante nasal como el menos reproducible tanto en sujetos normales (CV del 18\%) como en glaucomatosos (CV del 28\%) y el cuadrante inferior como el más reproducible (CV del 7\% en normales y $12 \%$ en glaucomas). Estos datos son superponibles a los nuestros, en que el cuadrante inferior resultó el más reproducible (CV del $2,9 \%$ en normales y $10,5 \%$ en glaucomas) y el temporal el menos (CV del 8,7\% en normales y $15,2 \%$ en glaucomas), con resultados similares en el superior (CV del 8,6\% en normales y $17,5 \%$ en glaucomas). Estos resultados son coherentes con la presencia de mayores espesores de la CFNR en los sectores inferiores y menores en los nasales y temporales.

Resultados similares fueron obtenidos por Gürses-Özden et al (16), que describieron el cuadrante inferior como el menos reproducible (CV del $16,5 \%$ al $19,6 \%$, según el explorador) y el espesor medio de la CFNR como el parámetro más reproducible. Dicho autor relaciona la mayor variabilidad de las mediciones en la zona nasal con la menor reflectancia de la luz que alcanza esa región por la posición del paciente y con la mayor presencia de vasos en esa zona.

Paunescu et al (17) calcularon mayor reproducibilidad si la exploración era realizada bajo midriasis farmacológica. En su población de referencia de sujetos normales observaron correlaciones mayores en los sectores superiores (Coeficiente de correla- ción intraclase, ICC, del 84\% en el correspondiente a las 11 horas y $85 \%$ a las 10 horas).

La diferencia en la reproducibilidad de exploraciones realizadas con fijación interna o externa fue calculada por Schuman et al (18), que mostraron mejores resultados en las llevadas a cabo mediante fijación interna. Del mismo modo, refirieron el sector nasal como el menos reproducible.

En cuanto a las diferencias de variabilidad detectadas al comparar grupos de sujetos normales con casos de glaucoma, la mayoría de los estudios refieren mayor variabilidad en el segundo grupo. Carpineto et al (19) observaron un coeficiente de correlación intraclase (ICC) significativamente mayor en sujetos normales que en glaucomas, determinando una variabilidad intrapersonal mayor en los pacientes con glaucoma. El mismo resultado fue obtenido por Blumenthal et al (15), que mostraron variabilidades intravisita e intersesión significativamente mayores en los casos de glaucoma que en sujetos normales.

A pesar de que la mayoría de los parámetros presentaron coeficientes de variación por encima del $10 \%$, el espesor medio de la CFNR mostró una buena reproducibilidad (CV 9,59\%). Éste parámetro ha sido referido por la mayoría de los autores como uno de los que presentan mayor rentabilidad diagnóstica en el glaucoma crónico simple $(20,21)$, lo cual justifica el uso de este instrumento en dicha enfermedad.

Nuestro trabajo refleja igualmente mayor variabilidad en los sujetos con glaucoma, a pesar de no alcanzar cifras estadísticamente significativas, que atribuimos a la mayor capacidad que ha demostrado tener el OCT para medir espesores de la CFNR mayores.

En general, podemos afirmar que la alta reproducibilidad del OCT hace de él un instrumento adecuado para el seguimiento de pacientes con hipertensión intraocular, al permitir relacionar cambios estructurales con aparición de neuropatía óptica o inicio del daño en la CFNR. En cuanto a su uso en pacientes con glaucoma, consideramos que se encuentra igualmente indicado, si bien existen ciertas limitaciones en la interpretación de ciertos parámetros dada su menor reproducibilidad.

\section{BIBLIOGRAFÍA}

1. Jonas JB, Fernandez MC, Sturmer J. Pattern of glaucomatous neuroretinal rim loss. Ophthalmology 1993; 100: 63-68. 
2. Polo V, Abecia E, Pablo LE, Pinilla I, Larrosa JM, Honrubia FM. Short-wavelength automated perimetry and retinal nerve fiber layer evaluation in suspects cases of glaucoma. Arch Ophthalmol 1998; 116: 1295-1298.

3. Sommer A, Katz J, Quigley HA, Millar NR, Robin AL, Richter $R C$, et al. Clinically detectable nerve fiber atrophy predeces the onset of glaucomatous field loss. Arch Ophthalmol 1991; 109: 77-83.

4. Heijl A, Lindgren GL, Olsson J, Asman P. Visual field interpretation with empiric probability maps. Arch Ophthalmol 1989; 107: 204-208.

5. Polo V, Larrosa JM, Pinilla I, Perez S, Gonzalvo F, Honrubia FM. Predictive value of short-wavelength automated perimetry: a 3-year follow-up study. Ophthalmology 2002; 109: 761-765.

6. Zangwill LM, Bowd C, Berry CC, Williams J, Blumenthal EZ, Sanchez-Galeana CA, et al. Discriminating between normal and glaucomatous eyes using the Heidelberg Retina Tomograph, GDx Nerve Fiber Analyzer, and Optical Coherence Tomograph. Arch Ophthalmol 2001; 119: 985993.

7. Hoh ST, Greenfield DS, Mistlberger A, Liebmann JM, Ishikawa H, Ritch R. Optical coherence tomography and scanning laser polarimetry in normal, ocular hypertensive, and glaucomatous eyes. Am J Ophthalmol 2000; 129: 129-135.

8. Pieroth L, Schuman JS, Hertzmark E, Hee MR, Wilkins $J R$, Coker J, et al. Evaluation of focal defects of the nerve fiber layer using optical coherence tomography. Ophthalmology 1999; 106: 570-579.

9. Guedes V, Schuman JS, Hertzmark E, Wollstein G, Correnti A, Mancini R, et al. Optical coherence tomography measurement of macular and nerve fiber layer thickness in normal and glaucomatous human eyes. Ophthalmology 2003; 110: 177-189.

10. Kanamori A, Nakamura M, Escano MF, Seya R, Maeda H, Negi A. Evaluation of glaucomatous damage on retinal nerve fiber layer thickness measured by optical coherence tomography. Am J Ophthalmol 2003; 135: 513-520.

11. Lederer DE, Schuman JS, Hertzmark E, Heltzer J, Velazques LJ, Fujimoto JC, et al. Analysis of macular volume in normal and glaucomatous eyes using optical coherence tomography. Am J Ophthalmol 2003; 135: 838-843.

12. Bowd C, Zangwill LM, Blumenthal EZ, Vasile C, Boehm $A G$, Gokhale PA, et al. Imaging of the optic disc and reti- nal nerve fiber layer: the effects of age, optic disc area, refractive error, and gender. J Opt Soc Am A Opt Image Sci Vis. 2002; 19: 197-207.

13. Gurses-Ozden R, Ishikawa H, Hoh ST, Liebmann JM, Mistlberger A, Greenfield DS, et al. Increasing sampling density improves reproducibility of optical coherence tomography measurements. J Glaucoma 1999; 8: 238241.

14. Mok KH, Lee VW, So KF. Increasing scans per examination improves the reproducibility on retinal nerve fiber layer measurements by optical coherence tomography. Optom Vis Sci 2004; 81: 268-271.

15. Blumenthal EZ, Williams JM, Weinreb RN, Girkin CA, Berry CC, Zangwill LM. Reproducibility of nerve fiber layer thickness measurements by use of optical coherence tomography. Ophthalmology 2000; 107: 2278-2282.

16. Gurses-Ozden R, Teng C, Vessani R, Zafar S, Liebmann $J M$, Ritch R. Macular and retinal nerve fiber layer thickness measurement reproducibility using optical coherence tomography (OCT-3). J Glaucoma 2004; 13: 238-244.

17. Paunescu LA, Schuman JS, Price LL, Stark PC, Beaton S, Ishikawa $H$, et al. Reproducibility of nerve fiber layer thickness, macular thickness, and optic nerve head measurements using StratusOCT. Invest Ophthalmol Vis Sci 2004: 45: 1716-1724.

18. Schuman JS, Pedut-Kloizman T, Hertzmark E, Hee MR, Wilkins JR, Coker JG, et al. Reproducibility of nerve fiber layer thickness measurements using optical coherence tomography. Ophthalmology 1996; 103: 1889-1898.

19. Carpineto P, Ciancaglini M, Zuppardi E, Falconio G, Doronzo E, Mastropasqua L. Reliability of nerve fiber layer thickness measurements using optical coherence tomography in normal and glaucomatous eyes. Ophthalmology 2003; 110: 190-195.

20. Kanamori A, Nagai-Kusuhara A, Escano MF, Maeda H, Nakamura M, Negi A. Comparison of confocal scanning laser ophthalmoscopy, scanning laser polarimetry and optical coherence tomography to discriminate ocular hypertension and glaucoma at an early stage. Graefes Arch Clin Exp Ophthalmol 2006; 244: 58-68.

21. Moreno-Montanes J, Alvarez-Vidal A, Sainz Gomez C, Rodriguez Conde R. Capa de fibras nerviosas en ojos glaucomatosos. Estudio realizado mediante OCT y su comparación con el campo visual. Arch Soc Esp Oftalmol 2002; 77: 435-441. 